\title{
Impacto femoroacetabular e lesão do lábio acetabular - Parte 1: Fisiopatologia e biomecânica
}

\section{Femoroacetabular Impingement and Acetabular Labral Tears - Part 1: Pathophysiology and Biomechanics}

\author{
Leandro Ejnisman ${ }^{10} \quad$ Walter Ricioli Júnior ${ }^{20}$ Marcelo Cavalheiro Queiroz ${ }^{10}$ \\ Jose Ricardo Negreiros Vicente ${ }^{10}$ Alberto Tesconi Croci ${ }^{10}$ Giancarlo Cavalli Polesello ${ }^{2(0)}$ \\ 1 Grupo de Quadril, Instituto de Ortopedia e Traumatologia, Hospital \\ das Clinicas, Faculdade de Medicina, Universidade de São Paulo, São \\ Paulo, SP, Brasil \\ ${ }^{2}$ Grupo de Quadril, Faculdade de Ciências Médicas, Santa Casa de São \\ Endereço para correspondência Leandro Ejnisman, MD, PhD, Rua \\ Dr. Ovídio Pires de Campos, 333, Cerqueira Cesar, São Paulo, \\ SP, 05403-010, Brasil (e-mail: leandro.ortopedia@gmail.com). \\ Paulo, São Paulo, SP, Brasil \\ Rev Bras Ortop 2020;55(5):518-522.

\begin{abstract}
Resumo
Palavras-chave

- quadril

- impacto femoroacetabular

- lábio acetabular

- medicina esportiva

- artroscopia de quadril

O impacto femoroacetabular (IFA) é uma importante causa de dor no quadril, e a principal etiologia da osteoartrose do quadril no jovem. O IFA é caracterizado por alterações sutis da anatomia do acetábulo e do fêmur proximal que podem causar lesões do complexo condrolabial. O lábio é uma estrutura fibrocartilaginosa essencial na estabilidade articular. Três tipos de IFA são descritos: came (onde há uma anesfericidade da cabeça femoral), pincer (onde há uma sobrecobertura acetabular) e misto (que apresenta características de ambos). A etiologia do IFA está relacionada com características genéticas e ambientais. O conhecimento desta doença e sua fisiopatologia é essencial para o tratamento de pacientes que apresentam dor no quadril.
\end{abstract}
Abstract
Keywords
- hip
- femoroacetabular impingement
- acetabular labrum
- sports medicine
- hip arthroscopy

Femoroacetabular impingement (FAl) is an important cause of hip pain, and the main etiology of hip osteoarthritis in the young population. Femoroacetabular impingement is characterized by subtle alterations in the anatomy of the acetabulum and proximal femur, which can lead to labrum tearing. The acetabular labrum is essential to the stability of the hip joint. Three types of FAI were described: cam (anespherical femoral head), pincer (acetabular overcoverage) and mixed (characteristics of both cam and pincer). The etiology of FAl is related to genetic and environmental characteristics. Knowledge of this condition is essential to adequately treat patients presenting with hip pain.

\section{Introdução}

A cirurgia do quadril avançou muito nas últimas 2 décadas. Diversas novas afecções que acometem a articulação coxo-femoral foram descritas, assim como novas técnicas de tratamento. Dentre estas afecções, o impacto femoroa-

recebido

29 de Setembro de 2019 aceito

12 de Novembro de 2019
DOI https://doi.org/

10.1055/s-0040-1702964. ISSN $0102-3616$ cetabular (IFA) e a lesão do lábio acetabular merecem destaque. O IFA é uma causa importante de dor no quadril do jovem, especialmente no esportista. Além disso, o IFA é a principal causa de osteoartrose do quadril (OA) no jovem.
Copyright $\odot 2020$ by Sociedade Brasileira License terms de Ortopedia e Traumatologia. Published by Thieme Revinter Publicações Ltda, Rio de Janeiro, Brazil 
O diagnóstico do IFA e da lesão labial são cada vez mais frequentes nos consultórios de ortopedia, e a artroscopia de quadril é um dos procedimentos ortopédicos que cresce mais rapidamente. Frente a este panorama, é imprescindível que o ortopedista conheça esta afecção e compreenda sua fisiopatologia.

\section{Histórico}

Em 1936, Smith-Petersen ${ }^{1}$ publicou pioneiramente sobre uma osteotomia femoral e acetabular utilizada no tratamento de pacientes com dor no quadril. Neste artigo, o autor questiona: "qual a origem da dor do paciente?", e responde: "o impacto entre o colo do fêmur e a parede anterior do acetábulo". A técnica cirúrgica consistia na exposição da articulação do quadril através de uma via tipo Smith-Petersen. Uma porção da parede anterior do acetábulo era ressecada, assim como uma porção da junção colo-cabeça femoral. Esta técnica é semelhante à descrita décadas depois para o tratamento cirúrgico do IFA, entretanto era utilizada em quadris já artrósicos.

Carlioz et al., ${ }^{2}$ em 1968, utilizaram pela primeira vez o termo came para descrever a deformidade femoral, neste caso associada a uma sequela de epifisiolistese. Em 1975, Stulberg et al., ${ }^{3}$ descrevem a deformidade em cabo de pistola, que estaria presente em $40 \%$ dos pacientes que desenvolvem OA de quadril.

Muitos anos se passariam até Klaue et al., ${ }^{4}$ em 1991, descreverem a síndrome do rebordo acetabular, na qual a displasia do quadril causa lesão do lábio acetabular, que seria precursora de OA. Reynolds et al., ${ }^{5}$ em 1999, demonstraram a associação entre a retroversão acetabular e a presença de dor no quadril; enquanto Myers et al., ${ }^{6}$ em 1999, reportaram cinco pacientes com dor após a realização de osteotomia periacetabular por impacto residual.

Todo este conhecimento acumulado por décadas culminou no artigo clássico de 2003, no qual Ganz et al., ${ }^{7}$ estabeleceram os conceitos do IFA moderno. Os autores sugerem que deformidades discretas femorais e acetabulares associadas ao movimento são responsáveis pelo desenvolvimento da OA, sugerem a classificação do IFA, achados clínicos e radiográficos desta doença, assim como seu tratamento por meio da luxação cirúrgica do quadril.

\section{Fisiopatologia}

O lábio acetabular é uma estrutura triangular fibrocartilaginosa localizada ao redor do rebordo acetabular, sendo interrompido inferiormente pelo ligamento transverso. O lábio foi o foco de diversos artigos recentes que demonstraram sua atuação na estabilidade articular. O lábio tem uma função importante no selo de sucção, responsável por manter uma pressão negativa dentro da articulação. Crawford et al., ${ }^{8}$ demonstraram que $43 \%$ menos força é necessária para distrair o quadril após ventilar a articulação (introdução de uma agulha entre o lábio e o acetábulo), em comparação com o estado intacto. Além disso, após a criação de uma lesão acetabular de $3 \mathrm{~mm}, 60 \%$ menos força foi necessária para criar a mesma distração. Estudos com elementos finitos e estudos biomecânicos em cadáveres demonstraram que a ressecção do lábio acetabular aumenta a taxa de consolidação da cartilagem. ${ }^{9,10}$ Consolidação é a compressão que ocorre na camada de cartilagem quando carga é aplicada. Estes estudos reforçam a idéia da função selante do lábio acetabular, que mantém um filme líquido entre o acetábulo e a cabeça femoral, que diminui a pressão sólido-sólido intra-articular.

O lábio acetabular apresenta grande resistência tênsil. Seu módulo de tensão, que é uma medida de sua rigidez e resistência tênsil ao alongamento, é 6 vezes maior do que o da borracha. ${ }^{11}$ Esta característica também é importante para sua função no selo de sucção do quadril. Por outro lado, o lábio acetabular não apresenta função importante no suporte da carga axial do quadril. O lábio suporta apenas de 1 a $2 \%$ da carga da articulação normal, subindo para 4 a $11 \%$ no quadril displásico. ${ }^{11-13}$

As estruturas cápsulo-ligamentares do quadril também são essenciais na estabilidade articular. Myers et al., ${ }^{14}$ demonstraram que o ligamento iliofemoral apresenta uma função importante ao limitar a rotação externa e a translação anterior do fêmur, e que o lábio acetabular apresenta uma função estabilizadora secundária nestes movimentos. A secção do ligamento iliofemoral aumentou a rotação externa do quadril (de $41,5^{\circ}$ para $54,4^{\circ}$ ), enquanto a secção do lábio acetabular não aumentou a rotação significativamente $\left(45,6^{\circ}\right)$. Outro achado importante deste estudo foi que o reparo do ligamento e do lábio restituíram a rotação inicial. Os autores sugerem que quando uma capsulotomia é realizada durante a artroscopia de quadril, esta deve ser reparada ao fim do procedimento.

\section{Classificação}

O IFA é dividido em 3 tipos: came, pincer ou misto ( - Figura 1). ${ }^{7,15} \mathrm{O}$ tipo pincer, também conhecido em português como

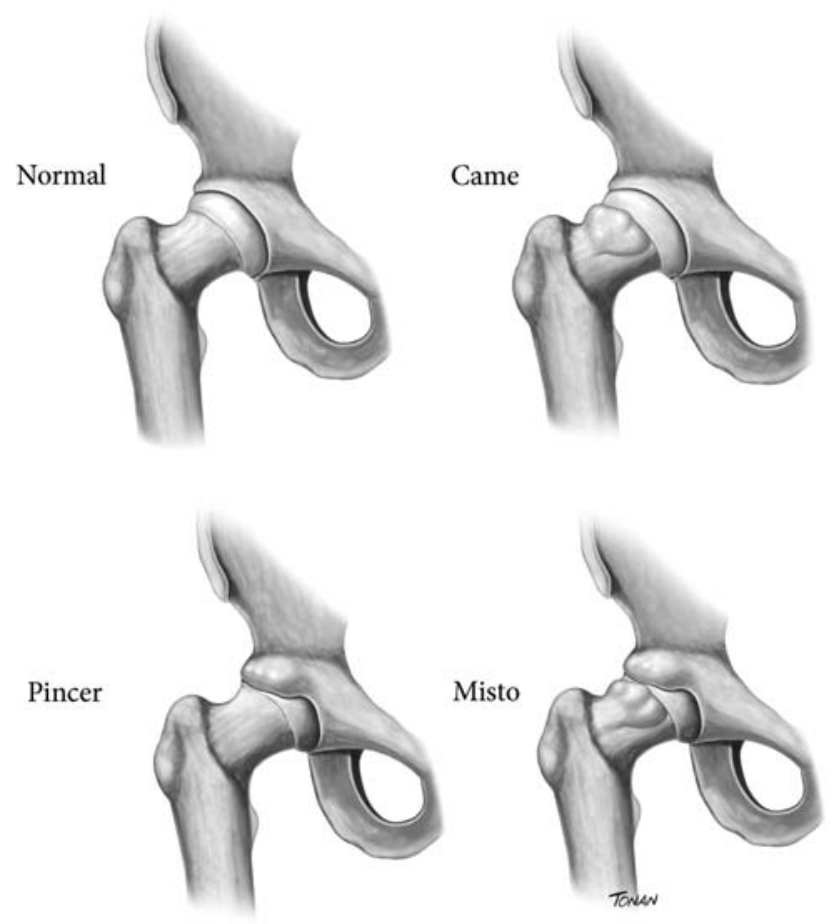

Fig. 1 Tipos de impacto femoroacetabular. 
torquês, é causado por uma alteração da cavidade acetabular. O acetábulo normal é antevertido, e recobre a cabeça femoral numa faixa estreita de normalidade. Tanto a falta de cobertura, que ocorre na displasia, quanto o excesso de cobertura que ocorre no pincer, são causa de dor e degeneração articular. No IFA tipo pincer há uma sobrecobertura do acetábulo em relação à cabeça femoral. Esta sobrecobertura pode ser focal em casos de retroversão acetabular ou global em casos de ângulo centro-borda aumentado. A lesão labial no pincer ocorre por um esmagamento do lábio entre o colo femoral e o rebordo acetabular. Por este motivo, o lábio no pincer encontra-se degenerado, podendo apresentar cistos intrasubstanciais. Outra lesão que pode ocorrer no pincer é a lesão por contragolpe. Neste caso, a cabeça femoral é alavancada posteriormente pela parede acetabular anterior proeminente, gerando uma lesão da cartilagem acetabular posterior.

O impacto tipo came é causado por uma anormalidade do fêmur. Para que o quadril tenha um movimento sem conflito, a cabeça femoral deve ser uma esfera perfeita. A deformidade do came ocorre na transição entre a cabeça e o colo femoral, gerando uma perda da esfericidade neste local. A lesão do lábio acetabular decorrente do came ocorre na transição entre a cartilagem acetabular e o lábio. À medida que a cabeça femoral anesférica adentra a cavidade acetabular, a cartilagem acetabular é avulsionada do lábio devido a uma força cisalhante entre a cabeça e a cartilagem. Por este motivo, lesões da cartilagem acetabular tipo carpete (cartilagem de aparência inicialmente sadia, porém descolada do osso subcondral) são comuns no came. O tecido labial nos estágios iniciais da lesão permanece sadio.

O terceiro tipo de IFA é o tipo misto, que apresenta uma associação das alterações pincer e came. 0 tipo misto é o mais frequente, com uma incidência de até $77 \%$ dos casos. ${ }^{16}$ Entretanto, mesmo no tipo misto, normalmente o paciente apresenta características mais predominantes de uma das morfologias came ou pincer.

Seldes et al., ${ }^{17}$ descreveram uma classificação histológica da lesão do lábio acetabular. 0 tipo 1 consiste num destacamento do lábio da cartilagem acetabular na transição entre o lábio fibrocartilaginoso e a cartilagem hialina acetabular. Já o tipo 2 consiste em um ou mais planos de clivagem dentro da substância acetabular. 0 tipo 2 está relacionado a ossificações endocondrais do lábio. Um artigo posterior modificou a classificação de Seldes e incluiu um lesão tipo 3, que possui características mistas, apresentando um destacamento do lábio associado e planos de clivagem na substância labial. ${ }^{18}$

\section{Etiologia}

O desenvolvimento do IFA é influenciado por características genéticas e ambientais. Pollard et al., ${ }^{19}$ estudaram irmãos de pacientes diagnosticados com IFA. Os irmãos de pacientes diagnosticados com came apresentaram um risco relativo 2,8 vezes maior de apresentar a mesma deformidade, enquanto irmãos de pacientes com deformidade tipo pincer apresentaram um risco relativo de 2,0. 0 grupo de irmãos também apresentou uma presença significativamente maior de OA de quadril grau 2 segundo a classificação Kellgren e Lawrence em comparação com o grupo controle (11 versus 0 ). Os autores concluem que há uma influência genética no desenvolvimento do IFA. Sekimoto et al., ${ }^{20}$ estudaram a associação entre polimorfismo genético e a sobrecobertura acetabular, e demonstraram que variações genéticas estão significativamente associadas à cobertura acetabular.

O desenvolvimento da deformidade came está relacionado a uma extensão lateral da fise de crescimento. ${ }^{21}$ Participação intensa em atividades esportivas durante a fase final da adolescência, próxima ao fechamento da fise, pode estar relacionada ao desenvolvimento do came, secundário a um estresse mecânico na fise da cabeça femoral. Murray et al., ${ }^{22}$ já em 1971 sugerem uma associação entre atividade esportiva na adolescência e OA de quadril na vida adulta. Os autores questionam se a OA de quadril idiopática teria mesmo etiologia indefinida, ou seria causada por alterações menores na articulação do quadril. Um estudo interessante avaliou a presença da deformidade came em atletas adolescentes de basquete em comparação com controles não atletas. ${ }^{23}$ Os controles apresentaram um ângulo alfa médio de $47,4^{\circ}$, enquanto os atletas apresentaram $60,5^{\circ}$. As diferenças foram ainda mais pronunciadas em fêmures com a fise já fechada. Os autores interpretaram este resultado como uma sugestão de que o came se desenvolveria durante o fechamento da fise. Uma pesquisa semelhante avaliou adolescentes jogadores de futebol pré-profissional. ${ }^{24}$ Um total de 63 atletas (média de 14,43 anos) foram acompanhados por no mínimo 2 anos. Um aumento significativo do ângulo alfa foi observado. A medida da extensão da fise de crescimento também foi associada ao ângulo alfa. Os autores também concluem que a deformidade came se desenvolve durante a maturação esquelética e estabiliza após o fechamento da fise. Uma revisão recente da literatura concluiu que atletas masculinos adolescentes envolvidos em hóquei no gelo, basquete e futebol, que treinam pelo menos três vezes por semana, apresentam maior risco de desenvolvimento de IFA. ${ }^{25}$

\section{Epidemiologia}

O consenso de Warwick sobre o IFA sugere o termo síndrome do IFA. ${ }^{26}$ o diagnóstico desta síndrome é baseado em sintomas clínicos, alterações de exame físico, e achados em exames de imagem. Esta consideração é importante porque diversos estudos epidemiológicos avaliam a presença de IFA em controles assintomáticos. A prevalência de alterações tipo IFA em controles é importante, porém vale a ressalva de que estes voluntários não apresentam a síndrome do IFA, por não possuírem sintomatologia no quadril.

Um revisão sistemática que avaliou a prevalência de deformidades tipo IFA em voluntários incluiu 26 estudos com mais de 2.000 pessoas ( $57,2 \%$ homens e $42,8 \%$ mulheres). ${ }^{27} \mathrm{O}$ ângulo alfa médio foi $54,1^{\circ}$, e a prevalência de came foi $37 \%$; porém, uma grande variação foi encontrada entre os estudos (7 a 100\% de prevalência). Uma grande diferença também foi notada quando comparando atletas e não atletas. Enquanto 54,8\% dos atletas apresentaram deformidade tipo came, apenas $23,1 \%$ dos não atletas apresentaram esta deformidade. Já a prevalência de deformidade tipo pincer foi $67 \%$. Sete estudos 
avaliaram a presença de lesão do lábio acetabular na ressonância magnética, e encontraram uma prevalência de 68,1\% de lesão.

A incidência de IFA e lesão labial sintomática em atletas não é conhecida. Estima-se que lesões do quadril são responsáveis por 5 a $6 \%$ das lesões esportivas. ${ }^{28,29}$ No esporte universitário americano, uma taxa de 53,06 lesões por 100.000 exposiçõesatleta foi reportada. ${ }^{30}$ Porém, estes estudos incluem lesões musculares na epidemiologia de lesões do quadril, não separando lesões articulares. Um estudo das lesões do futebol americano de 1997 a 2006 encontrou uma incidência de 3,1\% de lesões relacionadas ao quadril. ${ }^{31}$ Destas lesões, apenas $5 \%$ foram de causa articular. Outro estudo com atletas universitários de futebol americano universitários da $1^{\mathrm{a}}$ divisão avaliou a incidência de procedimentos cirúrgicos em um período de 10 anos (2004 a 2014). ${ }^{32}$ Foram identificados 254 procedimentos cirúrgicos, sendo que destes 15 cirurgias $(5,9 \%)$ foram reparos artroscópicos do lábio acetabular. Vale a ressalva que na última década ocorreu um grande aumento no conhecimento do IFA, e na difusão deste conhecimento entre os médicos do esporte. Portanto, é provável que esta incidência seja maior atualmente.

\section{Impacto Femoroacetabular e a Osteoartrose de Quadril}

A OA de quadril acomete $3 \%$ da população com mais de 30 anos nos Estados Unidos, onde mais de 200 mil próteses de quadril são realizadas por ano. ${ }^{33}$ No fim do dia, compreender a relação do IFA com a OA de quadril é um dos aspectos mais importantes no aconselhamento dos pacientes e na definição de estratégias de tratamento.

Agricola et al., ${ }^{34}$ realizaram um estudo prospectivo, conhecido como estudo CHECK, no qual mais de 1.000 pacientes foram acompanhados por 5 anos. Os pacientes realizaram uma radiografia de bacia, onde o ângulo alfa foi medido; e foram submetidos a exame físico. A presença de um came moderado (ângulo alfa $>60^{\circ}$ ) resultou num odds ratio (OR) de 3,67 de desenvolvimento de $\mathrm{OA}$, enquanto a presença de came grave (ângulo alfa $>83^{\circ}$ ) resultou num OR de 9,66 de desenvolvimento de $\mathrm{OA}$. A combinação came grave com diminuição de rotação interna $\left(<20^{\circ}\right)$ possuiu um valor preditivo positivo de $52,6 \%$ no desenvolvimento de OA grave do quadril ao fim do acompanhamento. Outro estudo com 1.003 pacientes do sexo feminino comparou radiografias de bacia com 20 anos de intervalo. ${ }^{35}$ A presença do came (definido como ângulo alfa $>65^{\circ}$ ) foi associada com o desenvolvimento de OA radiográfica. Para cada grau de aumento no ângulo alfa, ocorreu um aumento de $5 \%$ no risco de OA radiográfica e um aumento de $4 \%$ no risco de realização de artroplastia total de quadril.

A relação do IFA tipo pincer com o desenvolvimento de OA é menos claro. Outro estudo do grupo CHECK não encontrou relação entre ângulo centro-borda $>40^{\circ}$ e desenvolvimento de OA. ${ }^{36}$ Já Gosvig et al., ${ }^{37}$ encontraram um risco 2,4 vezes maior de desenvolvimento de $\mathrm{OA}$ em pacientes com ângulo centroborda $>45^{\circ}$. Vale lembrar que o ângulo centro-borda aumentado reflete uma sobrecobertura global acetabular. Estudos específicos sobre retroversão acetabular (sobrecobertura focal) sugerem uma possível correlação com a OA de quadril.
Kim et al., ${ }^{38}$ encontraram uma correlação entre diminuição do espaço articular e a retroversão acetabular em um estudo com tomografias computadorizadas de pacientes submetidos ao exame por motivos não ortopédicos. Giori et al., ${ }^{39}$ realizaram um estudo caso-controle, comparando radiografias de pacientes submetidos a prótese total de quadril por OA e controles assintomáticos. Uma incidência de $20 \%$ de retroversão acetabular foi encontrada nos pacientes em comparação com uma incidência de 5\% nos controles. Os autores concluem que há uma relação entre retroversão acetabular e OA de quadril.

O estudo da relação entre o IFA e a OA de quadril é um campo em expansão e que necessita de mais pesquisa. $\mathrm{Na}$ prática clínica, notamos que alguns pacientes portadores de IFA apresentam uma rápida evolução para OA, enquanto outros apresentam uma evolução bastante lenta. Compreender quais pacientes apresentam risco alto de progressão e quais pacientes estão menos suscetíveis será um grande avanço que acarretará uma melhora no tratamento do paciente portador de IFA.

\section{Considerações Finais}

O IFA é uma síndrome caracterizada por dor no quadril associada a alterações sutis na anatomia da articulação coxo-femoral. O IFA é uma causa importante de lesão do lábio acetabular, que é uma estrutura essencial na biomecânica do quadril. O conhecimento desta doença é de suma importância para o ortopedista que trata pacientes com dor no quadril.

Conflito de Interesses

Os autores declaram não haver conflito de interesses.

\section{Agradecimentos}

Os autores gostariam de agradecer Rodrigo Tonan pela ilustração médica deste artigo.

\section{Referências}

1 Smith-Petersen MN. Treatment of malum coxae senilis, old slipped upper femoral epiphysis, intrapelvic protrusion of the acetabulum, and coxa plana by means of acetabuloplasty. J Bone Joint Surg Am 1936;18:869-880

2 Carlioz H, Pous JG, Rey JC. [Upper femoral epiphysiolysis]. Rev Chir Orthop Repar Appar Mot 1968;54(05):387-491

3 Stulberg SD, Cordell LD, Harris WH, Ramsey PL, MacEwan GD. Unrecognized childhood hip disease: a major cause of idiopathic osteoarthritis of the hip. In: The Hip. Proceedings of The Third Meeting of the Hip Society. St. Louis, MOC.V. Mosby 1975

4 Klaue K, Durnin CW, Ganz R. The acetabular rim syndrome. A clinical presentation of dysplasia of the hip. J Bone Joint Surg Br 1991;73(03):423-429

5 Reynolds D, Lucas J, Klaue K. Retroversion of the acetabulum. A cause of hip pain. J Bone Joint Surg Br 1999;81(02):281-288

6 Myers SR, Eijer H, Ganz R. Anterior femoroacetabular impingement after periacetabular osteotomy. Clin Orthop Relat Res 1999; (363):93-99

7 Ganz R, Parvizi J, Beck M, Leunig M, Nötzli H, Siebenrock KA. Femoroacetabular impingement: a cause for osteoarthritis of the hip. Clin Orthop Relat Res 2003;(417):112-120

8 Crawford MJ, Dy CJ, Alexander JW, et al. The 2007 Frank Stinchfield Award. The biomechanics of the hip labrum and the stability of the hip. Clin Orthop Relat Res 2007;465(465):16-22 
9 Ferguson SJ, Bryant JT, Ganz R, Ito K. The acetabular labrum seal: a poroelastic finite element model. Clin Biomech (Bristol, Avon) 2000;15(06):463-468

10 Ferguson SJ, Bryant JT, Ganz R, Ito K. An in vitro investigation of the acetabular labral seal in hip joint mechanics. J Biomech 2003; 36(02):171-178

11 Bsat S, Frei H, Beaulé PE. The acetabular labrum: a review of its function. Bone Joint J 2016;98-B(06):730-735

12 Konrath GA, Hamel AJ, Olson SA, Bay B, Sharkey NA. The role of the acetabular labrum and the transverse acetabular ligament in load transmission in the hip.J Bone Joint Surg Am 1998;80(12):1781-1788

13 Henak CR, Ellis BJ, Harris MD, Anderson AE, Peters CL, Weiss JA. Role of the acetabular labrum in load support across the hip joint. J Biomech 2011;44(12):2201-2206

14 Myers CA, Register BC, Lertwanich P, et al. Role of the acetabular labrum and the iliofemoral ligament in hip stability: an in vitro biplane fluoroscopy study. Am J Sports Med 2011;39(Suppl):85S-91S

15 Lavigne M, Parvizi J, Beck M, Siebenrock KA, Ganz R, Leunig M. Anterior femoroacetabular impingement: part I. Techniques of joint preserving surgery. Clin Orthop Relat Res 2004;(418):61-66

16 Philippon MJ, Briggs KK, Yen YM, Kuppersmith DA. Outcomes following hip arthroscopy for femoroacetabular impingement with associated chondrolabral dysfunction: minimum two-year follow-up. J Bone Joint Surg Br 2009;91(01):16-23

17 Seldes RM, Tan V, Hunt J, Katz M, Winiarsky R, Fitzgerald RH Jr. Anatomy, histologic features, and vascularity of the adult acetabular labrum. Clin Orthop Relat Res 2001;(382):232-240

18 Ejnisman L, Domb BG, Souza F, Junqueira C, Vicente JRN, Croci AT. Are femoroacetabular impingement tomographic angles associated with the histological assessment of labral tears? A cadaveric study. PLoS One 2018;13(06):e0199352

19 Pollard TC, Villar RN, Norton MR, et al. Genetic influences in the aetiology of femoroacetabular impingement: a sibling study. J Bone Joint Surg Br 2010;92(02):209-216

20 SekimotoT, Kurogi S, Funamoto T, et al. Possible association of single nucleotide polymorphisms in the 3' untranslated region of HOXB9 with acetabular overcoverage. Bone Joint Res 2015;4(04):50-55

21 Siebenrock KA, Wahab KH, Werlen S, Kalhor M, Leunig M, Ganz R. Abnormal extension of the femoral head epiphysis as a cause of cam impingement. Clin Orthop Relat Res 2004;(418):54-60

22 Murray RO, Duncan C. Athletic activity in adolescence as an etiological factor in degenerative hip disease. J Bone Joint Surg Br 1971;53(03):406-419

23 Siebenrock KA, Ferner F, Noble PC, Santore RF, Werlen S, Mamisch TC. The cam-type deformity of the proximal femur arises in childhood in response to vigorous sporting activity. Clin Orthop Relat Res 2011;469(11):3229-3240

24 Agricola R, Heijboer MP, Ginai AZ, et al. A cam deformity is gradually acquired during skeletal maturation in adolescent and young male soccer players: a prospective study with minimum 2-year follow-up. Am J Sports Med 2014;42(04):798-806
25 de Silva V, Swain M, Broderick C, McKay D. Does high level youth sports participation increase the risk of femoroacetabular impingement? A review of the current literature. Pediatr Rheumatol Online J 2016;14(01):16

26 Griffin DR, Dickenson EJ, O'Donnell J, et al. The Warwick Agreement on femoroacetabular impingement syndrome (FAI syndrome): an international consensus statement. Br J Sports Med 2016;50(19): 1169-1176

27 Frank JM, Harris JD, Erickson BJ, et al. Prevalence of Femoroacetabular Impingement Imaging Findings in Asymptomatic Volunteers: A Systematic Review. Arthroscopy 2015;31(06):1199-1204

28 Keogh MJ, Batt ME. A review of femoroacetabular impingement in athletes. Sports Med 2008;38(10):863-878

29 Prather H, Colorado B, Hunt D. Managing hip pain in the athlete. Phys Med Rehabil Clin N Am 2014;25(04):789-812

30 Kerbel YE, Smith CM, Prodromo JP, Nzeogu MI, Mulcahey MK. Epidemiology of Hip and Groin Injuries in Collegiate Athletes in the United States. Orthop J Sports Med 2018;6(05):2325967118771676

31 Feeley BT, Powell JW, Muller MS, Barnes RP, Warren RF, Kelly BT. Hip injuries and labral tears in the national football league. Am J Sports Med 2008;36(11):2187-2195

32 Mehran N, Photopoulos CD, Narvy SJ, Romano R, Gamradt SC, Tibone JE. Epidemiology of Operative Procedures in an NCAA Division I Football Team Over 10 Seasons. Orthop J Sports Med 2016;4(07):2325967116657530

33 Nho SJ, Kymes SM, Callaghan JJ, Felson DT. The burden of hip osteoarthritis in the United States: epidemiologic and economic considerations. J Am Acad Orthop Surg 2013;21(Suppl 1):S1-S6

34 Agricola R, Heijboer MP, Bierma-Zeinstra SM, Verhaar JA, Weinans $\mathrm{H}$, Waarsing JH. Cam impingement causes osteoarthritis of the hip: a nationwide prospective cohort study (CHECK). Ann Rheum Dis 2013;72(06):918-923

35 Thomas GE, Palmer AJ, Batra RN, et al. Subclinical deformities of the hip are significant predictors of radiographic osteoarthritis and joint replacement in women. A 20 year longitudinal cohort study. Osteoarthritis Cartilage 2014;22(10):1504-1510

36 Agricola R, Heijboer MP, Roze RH, et al. Pincer deformity does not lead to osteoarthritis of the hip whereas acetabular dysplasia does: acetabular coverage and development of osteoarthritis in a nationwide prospective cohort study (CHECK). Osteoarthritis Cartilage 2013;21(10):1514-1521

37 Gosvig KK, Jacobsen S, Sonne-Holm S, Palm H, Troelsen A. Prevalence of malformations of the hip joint and their relationship to sex, groin pain, and risk of osteoarthritis: a populationbased survey. J Bone Joint Surg Am 2010;92(05):1162-1169

38 Kim WY, Hutchinson CE, Andrew JG, Allen PD. The relationship between acetabular retroversion and osteoarthritis of the hip. J Bone Joint Surg Br 2006;88(06):727-729

39 Giori NJ, Trousdale RT. Acetabular retroversion is associated with osteoarthritis of the hip. Clin Orthop Relat Res 2003;(417): 263-269 